\title{
Long Term Evolution (LTE) Handover Parameter Optimation
}

\author{
Maksum Pinem ${ }^{1}$, Dhara Monica Aqsa ${ }^{2}$, Emerson P. Sinulingga ${ }^{3}$ and Suherman ${ }^{4}$ \\ \{maksum@usu.ac.id ${ }^{1}$, dharamonica@gmail.com ${ }^{2}$ \} \\ 1,2,3,4Electrical Engineering Department, Universitas Sumatera Utara, Indonesia
}

\begin{abstract}
Handover is one of the facilities in the cellular communication system to ensure the continuity of communication when the customer moves from one cell to another cell. In this research will be analyzed on the optimization of soft handover performance of three eNB on LTE network with threshold method. Network performance parameters observed were RSRP (Reference Signal Received Power) and number of handover. The simulation result shows that with thershold value change we get RSRP value which is more optimal for the three eNB, each of three eNB are distanced by 8000 $\mathrm{m}$. When the thershold value is $-113 \mathrm{dBm}$ at a speed of $15 \mathrm{~m} / \mathrm{s}$, the RSRP value is very much greater than the threshold value so can be to minimize the number of handovers. Also it is found that the speed effect on the number of handover, where the greater the speed then the number of handover will be even greater.
\end{abstract}

Keywords: Handover, Threshold, LTE.

\section{Introduction}

The long term evolution (LTE) is an advance cellular communication system that is part of the third generation partnership project (3GPP) release 8 to enhance the UMTS standard. LTE is able to cover wider area and deliver higher rate services, reduce the operational cost and support multiple antenna technologies. LTE is also flexible in bandwidth management and interoperable with the existing technologies (Sauter, 2011). LTE is comparable to worldwide interoperability microwave access (WiMAX) technologies in broadband wireless access (Marwan Al-Akaidi, 2015).

The handover is well supported to enable mobility between one to other e Node B (eNB)(Wardana Lingga, 2010).The soft handover and the hard handover are both available in LTE. Hard handover uses break before make, while soft handover is make before break(Mittal, P., Darwazeh, I., Manukyan, 2014). Some soft handover teqhniques have been proposed and can be found in (Jouli, Imen., Frikha, M., Tousin, 2016). In order to make sure that handover occurs smoothly, signal degradation and power threshold should be determined precisely (Wardana Lingga, 2010). Soft handover is preferred than the hard one as communication disconnection is less frequently (Halgamuge, 2006). Signal strength variations caused propagation and threshold for handover decision should be taken into consideration carefully.

The handover has a lot to do with switching loads in cellular networks, so handover events need to be assessed to determine the optimal parameters.Some parameters to examine the optimal handover method are: the signal level that MS deserves, the expected number of handover, the number of unnecessary handover and the failed handover event. Selection of handover methods is needed to reduce the cost of radio transmission sources while maintaining Quality of Service (QoS) standards. Signal received MS consists of three components, namely: path loss, shadow fading and fast fading (Halgamuge, 2006). 
In this research is used soft handover method to find out how the process of handover parameter optimization on LTE network, how the algorithm of the soft handover and what parameters influence from the performance of the soft handover algorithm. The problem in this research is how to get optimal value based on handover parameters in LTE network. This paper examines soft handover performances based onnumber of handover andreference signal received power(RSRP). In conducting this research, the authors have examined several similar studies that have been done by previous researchers as a reference and comparison to complete the process of this study.

\section{Method}

In order to evaluate the soft handover performances, the RSS based handover on LTE is modelled on Mat lab. Link budget, criteria and network parameters are set at first place.

The evaluated network consists of three eNB. User UE moves from $\mathrm{eNB}_{1}$ toeNB 2 and fromeNB ${ }_{2}$ to $\mathrm{eNB}_{3}$, which is illustrated in Figure 1.

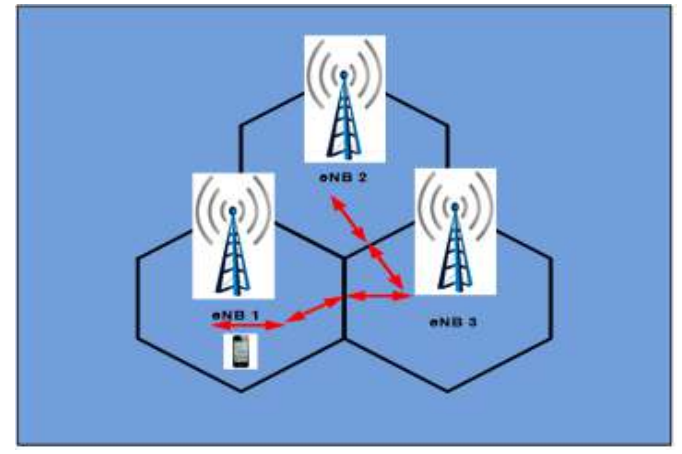

Fig. 1. LTE Movement model.

The illustration movement model to be used on the LTE network will be shown in Figure 1.

1. These three eNBs will also have the same transmission power as the distance and speed that has been determined.

2. The UE will receive a strong signal with an interval-determined distance.

3. There are three parameters to be observed in this researchare: RSRP, active set and number of handover.

4. The soft handover initiation model used is the value based on RSS (Received Signal Strength) based handover that is the accepted pilot signal strength and the soft handover algorithm model used is the threshold algorithm.

5. Simulation results with 1 time iteration starting at a distance of 0 meters up to a distance of 8000 meters. Starting from eNB1 to eNB3 on an LTE network. In this simulation eNB will show the serving and eNB handover.

The path distance ismodelled by using Equation1. In order to determine $\mathrm{x}_{1}, \mathrm{y}_{1}, \mathrm{x}_{2}$ and $\mathrm{y}_{2}$, Equation (2) and Equation (3) are employed.

$$
\mathrm{r}=\sqrt{\left(x_{1}-x_{2}\right)^{2}+\left(y_{1}-y_{2}\right)^{2}}
$$




$$
\begin{array}{cc}
x_{1}=\left(\frac{d x \cos \theta x t}{t_{\text {tempuh }}}\right) & y_{1}=-\tan \theta \mathrm{xX}_{1} \\
x_{2}=x_{E N B}-x_{1} & \mathrm{y}_{2}=y_{E N B}-\mathrm{y}_{1}
\end{array}
$$

Where, $\mathrm{x}_{1}$ (coordinate $\mathrm{x}$ UE), $\mathrm{y}_{1}$ (coordinate $\mathrm{y}$ UE ), $\mathrm{x}_{2}$ (coordinate $\mathrm{x}$ eNB)dan $\mathrm{y}_{2}$ (coordinate $\mathrm{y} e \mathrm{NB}$ ). To determine $\mathrm{x}_{1}$ and $\mathrm{y}_{1}$ also $\mathrm{x}_{2}$ and $\mathrm{y}_{2}$ are respectively shown in Equations (2) and Equation (3).

The three eNB have the same transmitting power and uses received signal strength (RSS) based handover with hysteresis-threshold based soft handover.

The maximum path loss(MAPL) is determined by using link budget parameter shown in Table 1, as follow:

Table 1. Link Budget.

\begin{tabular}{cc} 
Table 1. Link Budget. \\
\hline Parameter & LTE \\
\hline Transmitter - eNB & \\
Tx Power (dBm) & 46 \\
Tx AntennaGain (dBi) & 18 \\
Cable Loss (dB) & 2 \\
EIRP (dBm) & 62 \\
Receiver - UE & \\
UE Noise Figure (dB) & 7 \\
Thermal Noise (dB) & $-104,5$ \\
Receiver Noise Floor (dBm) & $-97,5$ \\
SINR(dB) & -9 \\
Receiver Sensitivity (dBm) & $-106,5$ \\
Interference Margin (dB) & 4 \\
Control Channel Overhead (\%) & 20 \\
Rx Antenna Gain (dBi) & 0 \\
Body Loss (dB) & 0 \\
Maximum Path Loss & 163,5 \\
\hline
\end{tabular}

The propagation model uses Erceg Greenstein as shown in Equation (4). $R_{\mathrm{m}}=10 \frac{\text { MAPL }-80,76-15,81-6 \log \left(\frac{\mathrm{f}}{2000}\right)-\left(-10,8 \log \left(\frac{\mathrm{ht}}{2}\right)\right)}{43,75} \times 100$

The LTE eNB coverage is shown in Table 2.

Table 2. eNB coverage.

\begin{tabular}{lc}
\multicolumn{2}{c}{ Table 2. eNB coverage. } \\
\hline \multicolumn{1}{c}{ Parameter } & Value \\
\hline Frequency (MHz) & 2600 \\
\hline $\begin{array}{l}\text { High Effective Antenna } \\
\text { eNB/ ht (m) }\end{array}$ & 18 \\
\hline High Antenna UE/ hr $(\mathrm{m})$ & 2 \\
\hline Shadow Fading / terrain type B(dB) & 62 \\
\hline
\end{tabular}


In LTE networks decisions on handover are made by UE or network based on the quality of links. In LTE, the UE will make the final decision to determine the occurrence of the handover or not, while the eNB makes recommendations on the target eNB candidate for the handover. Handover decisions are based on the best signal quality of UE reported results periodically.

The user UE decides handover based on eNB recommendation where the best signal is. The RSSP is used to determinecell selection, cell reselection, andhandover. RSRP is measured based on Equation (5) (Sainju, P. M., 2002).

$\mathrm{RSRP}=$ EIRP - Pathloss $t o U E+$ ShadowFading $(\sigma=\mathfrak{I})$

Where, RSRP $(\mathrm{dBm})$ : power received UE from eNB at a distance $\mathrm{d}_{0}$, EIRP $(\mathrm{dBm})$ : power output from the antenna (Tx_power + gain_antenna- losscable), Shadow fading $(\mathrm{dB})$ : large scale fading that distributes lognormal.

The shadow fading and pathloss is computed by using Equation (6) and Equation (7) (Chen, Y., 2003).

ShadowFading $=\frac{e-\frac{\left[(M-m)^{2}\right]}{2 \sigma}}{3(2 \pi)^{1 / 2}}$

Pathloss $=A+(10 \cdot \alpha \cdot \log (d / 100))+$ Shadowing Fading

$$
+\mathrm{Kf}+\mathrm{Kr}
$$

Where, $\mathrm{M}$ : normal random variable strong signal $(\mathrm{dBm}), \mathrm{m}$ : normal random variable strong signal $(\mathrm{dBm}), \mathrm{A}:$ track attenuation $(\mathrm{dB}), \mathrm{K}_{\mathrm{f}}$ : frequency correction factor, $\mathrm{K}_{\mathrm{r}}$ : factor correctiondevice $\mathrm{Rx}, \alpha$ : pathloss exponent, $\sigma$ : standard deviation.

The handover performances are determined by:

a. The number of handover events $U_{k}(l)$ on the path 1 consisting of $\mathrm{N}$ point sampled signal is expessed by the following Equestion (8)(Pinem, Maksum and all, 2017)

$$
U_{k}(l)=\sum_{k=1}^{N-1} U_{k}
$$

Where, $U_{k}=1$ in case of handover, otherwise $U_{k}=0$ stated that handover does not happen handover.The handover average value of the s number of 1 trajectory is written by following Equation (9)

$$
\overline{\text { Handover }}=\sum_{l=1}^{s} \frac{U_{k}(l)}{s}
$$

b. Active set changes based on the following conditions(Singh, N.P., Brahmijt Singh, 2010):

$\hat{\mathrm{S}}_{1}>\hat{\mathrm{S}}_{2}+$ Hyst $_{\mathrm{ADD}} \rightarrow \mathrm{U}_{\mathrm{k}}=0$

$\hat{\mathrm{S}}_{1}<\hat{\mathrm{S}}_{2}+$ Hyst $_{\mathrm{ADD}} \mid \hat{\mathrm{S}}_{2}>\hat{\mathrm{S}}_{1}$ 
+ Hyst $_{\text {DROP }} \rightarrow \mathrm{U}_{\mathrm{k}}=1$

$\mathrm{NO}_{\text {update }}=$ number of

transitionvalues $\mathrm{U}_{\mathrm{k}}$

c. The outage occurs if the following probability occurs Equation (13) :

$$
\mathrm{P}_{\mathrm{o}}(\mathrm{d})=\mathrm{Q}\left(\hat{\mathrm{S}}_{\text {best }}-\mathrm{S}_{\mathrm{min}} / \sigma\right)
$$

d. The Soft Handover Region (SHR) ratio is the ratio of the soft handover area to the cell area. The SHR equation is seen in Equation (14).

$$
\mathrm{SHR}=1-\left(\mathrm{R}_{\mathrm{h}} / \mathrm{R}_{\mathrm{s}}\right)^{2}
$$

UE is assumed to move straight from eNB1 to eNB 2, then from eNB 2 to eNB3. The three eNBs are separated by distance (d). This simulation looks at the performance of a moving user from LTE cells to LTE cells, with input parameters are threshold, hysteresis ADD and DROP, UE distance to eNB, sampling time, UE speed and number of iterations.For each eNB are given different inputs such as MAPL, frequency, EIRP, high antenna and high eNB adapted to the network specification of the system model.The output of the simulation will be observed are RSRP (signal strength on the LTE network), active set, handover decision, iteration, travel time, sample point, and network conditions.

\section{Results}

Figure 2 shows the RSRP signal strength of three eNBs. When UE moves as far as 3333.3 $\mathrm{m}$ with threshold $=-100 \mathrm{dBm}$, UE experiences handover from eNB1 to eNB2, since the signal strength coming from eNB1 is $-120 \mathrm{dBm}$ which is smaller than threshold value, whereas the signal strength coming from NB2 is $-99 \mathrm{dBm}$ whose value is greater than the threshold value

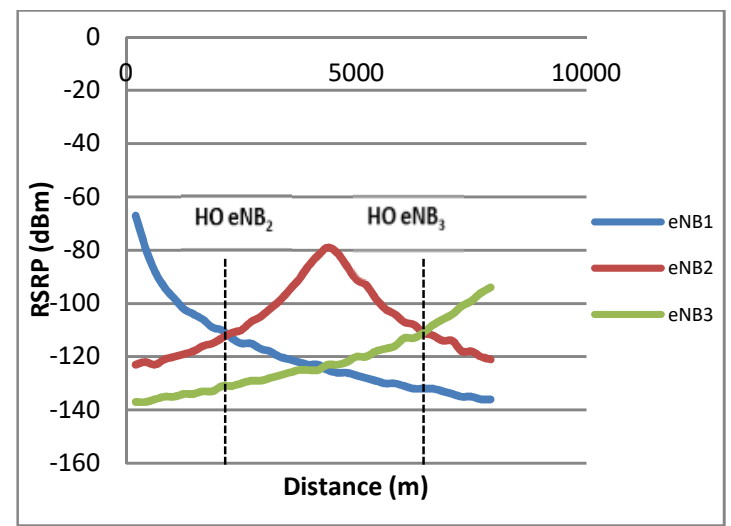

Fig. 2. The RSRP value graph for the distance at threshold $=-100 \mathrm{dBm}$ on all three eNB.

When the UE is at a distance of $7500 \mathrm{~m}$, UE experiences a handover to eNB3, where the signal strength obtained by eNB2 is $-118 \mathrm{dBm}$ and this value is smaller than the threshold value while the signal strength value in eNB3 is $-99 \mathrm{dBm}$ and this value is larger than the threshold value. 
In Figure 3, is shown again RSRP values received by UE against the distance at threshold value of $-100 \mathrm{dBm}$, can be seen that at $3333.3 \mathrm{~m}$ distance there is handover (HO) to eNB2 and at $7500 \mathrm{~m}$ distance occurs handover to eNB3. So, the number of handovers that occurs as much as 2 times when speed $5 \mathrm{~m} / \mathrm{s}$.

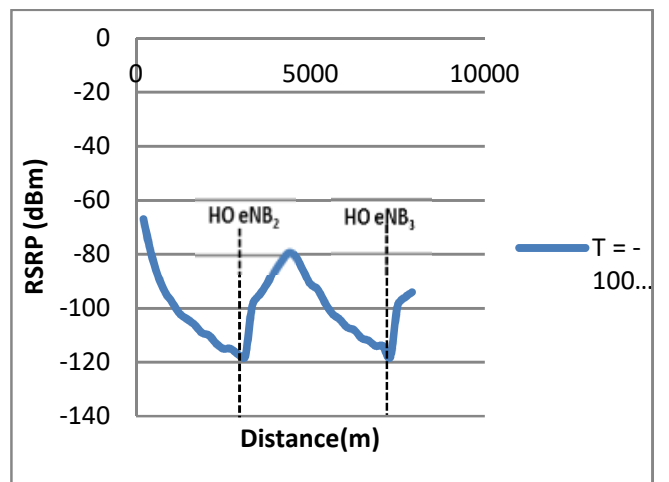

Fig. 3. Graph of RSRP values received by UE against distance at threshold $=-100 \mathrm{dBm}$.

In Figure 4 shown the RSRP value of the against distance for vary of threshold value ($100 \mathrm{dBm},-105 \mathrm{dBm},-110 \mathrm{dBm},-115 \mathrm{dBm})$. It can be seen that to obtain the optimal threshold value is at a value of $-110 \mathrm{dBm}$ to $-115 \mathrm{dBm}$, but at threshold value $=-115 \mathrm{dBm}$, eNB can only be handled until eNB2.

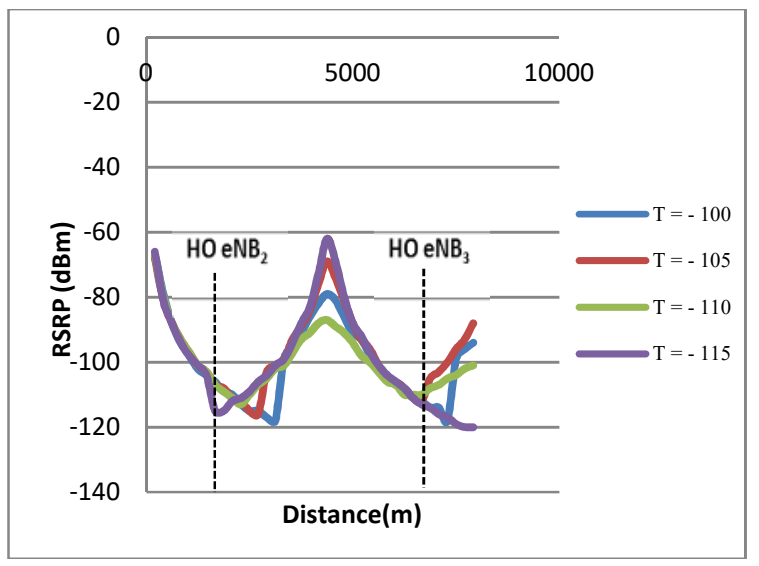

Fig. 4. Graph of RSRP value to distance at time of threshold change.

From Figure 5, it can be determined that the optimal threshold value for a range of threshold values, is from $-110 \mathrm{dBm}$ to $-114 \mathrm{dBm}$. Where the optimum threshold value obtained is at the value of threshold $=-113 \mathrm{dBm}$ with $\mathrm{RSRP}$ value $=-62 \mathrm{dBm}$ at distance $=$ $4375 \mathrm{~m}$, with the number of handover that occurs 4 times at the speed of $15 \mathrm{~m} / \mathrm{s}$. Based on RSRP Value received by EU in threshold $=-113 \mathrm{dBm}$, then at 2,500 $\mathrm{m}$ distance happened handover to eNB2 and at distance $6875 \mathrm{~m}$ happened handover to eNB3. 


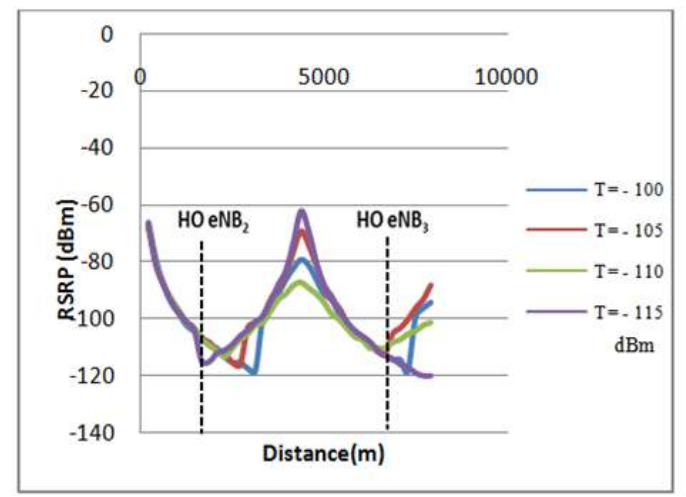

Fig, 5. Graph of RSRP value to distance at time of threshold change.

Figure 6 shows the change of RSRP values occurring on the LTE network for speed changes of $5,10,15,20,25$ and $30 \mathrm{~m} / \mathrm{s}$ for a fixed distance of $8000 \mathrm{~m}$. It can be observed that the RSRP values at each velocity of $5,10,15,20,25$ and $30 \mathrm{~m} /$ are respectively - 99, -91 , $-79,-88,-85$ and $-89 \mathrm{dBm}$.

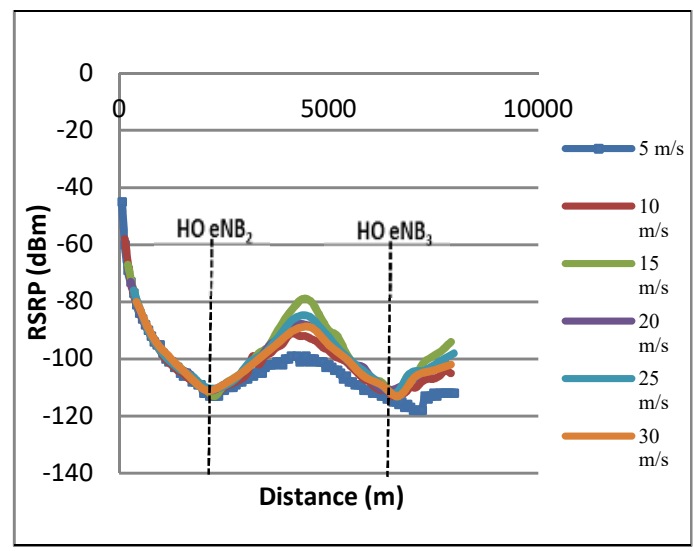

Fig. 6. Graph of RSRP value to distance at time of change of speed.

At the speed of $15 \mathrm{~m} / \mathrm{s}$ and the acceptance value of RSRP - $79 \mathrm{dBm}$, then at a distance of 2,500 m occurred handover to eNB2 and at $6875 \mathrm{~m}$ distance occurred handover to eNB3.

The observation results on the number of handover to change the value of the speed can be seen in Figure 7. At the time of $5 \mathrm{~m} / \mathrm{s}$ the number of handover that occurs as much as 3 times and also obtained that the greater the speed then the number of handover will be greater. 


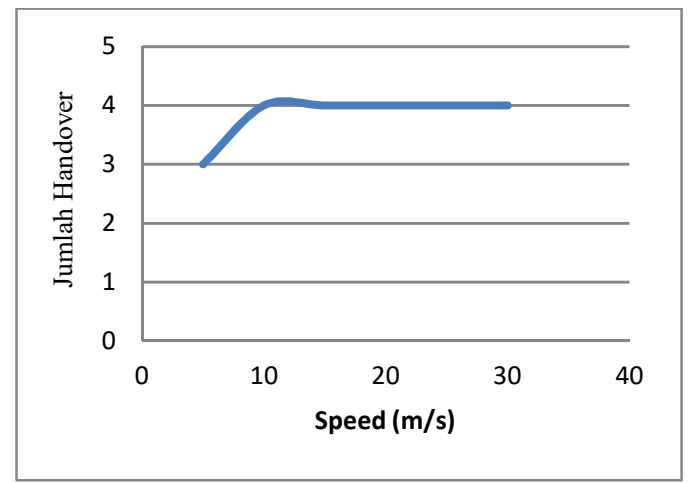

Fig. 7. Graph of RSRP value to distance at time of change of speed.

\section{Conclusions}

It has been shown that RSRP values changes as UE moves. The change on threshold results different numbers of handover. This RSRP increment is potentially decreasing the number of handover, which indicates the better performance. In this evaluation, the simulation result shows that with threshold value change, obtained RSRP value which is more optimal for the three eNB, each of three eNB are distanced by $8000 \mathrm{~m}$. When the thershold value is -113 $\mathrm{dBm}$ at a speed of $15 \mathrm{~m} / \mathrm{s}$, the RSRP value is very much greater than the threshold value so can be to minimize the number of handovers. Also it is found that the speed effect on the number of handover, where the greater the speed then the number of handover will be even greater.

\section{References}

[1] Halgamuge, M. N. (2006). Performance Evaluation and Enhancement of Mobile and Sensor Networks. In -.

[2] Jouli, Imen., Frikha, M., Tousin, H. (2016). An extended soft handover scheme for real-time service with QoS guarantee in LTE-A system. IEEE.

[3] Marwan Al-Akaidi, S. (2015). A transport layer protocol for uplink WiMAX video streaming. International Journal of Multimedia and Ubiquittous Engineering, 10(1), $19-32$.

[4] Mittal, P., Darwazeh, I., Manukyan, H. (2014). Overhead Estimation During Intra eNB Handover in 4G LTE Systems. In IEEE 9th International Symposium on Communication Systems, Networks \& Digital Sign (CSNDSP).

[5] Sainju, P. M. (2002). LTE Performance Analysis on 800 and $1800 \mathrm{MHz}$ Bands. Tampere University of Technology.

[6] Sauter, M. (2011). From GSM to LTE an introduction to mobile networks and mobile broadband. Wley Inc.

[7] Wardana Lingga, N. M. (2010). Teknologi wireless communication dan wireless broadband. Jogjakarta: Penerbit Andi. 